\title{
Binary star formation from ring fragmentation
}

\author{
D. A. Hubber and A. P. Whitworth
}

\author{
School of Physics \& Astronomy, Cardiff University, 5 The Parade, Cardiff CF24 3YB, UK \\ e-mail: david.hubber@astro.cf.ac.uk
}

Received 24 November 2004 / Accepted 11 March 2005

\begin{abstract}
We present a simple model of binary star formation based on the assumption that rotating prestellar cores collapse to form rings and these rings then fragment into protostars. We assume that each ring spawns a small number $(\mathcal{N} \leq 6)$ of protostars, and that the condensation of the protostars is sufficiently rapid that they can subsequently be treated as point masses. The first part of the model is therefore to simulate the dynamical evolution of a ring of $\mathcal{N}$ stars and record the properties of the single stars, binaries and higher multiples that form as a result of the dissolution of the ring. The masses of the individual stars in a ring are drawn from a $\log$-normal distribution with dispersion $\sigma_{\log M}$. This part of the model is perfomed for many different realizations of the ring, to obtain good statistics. It can be formulated using dimensionless variables and immediately yields the overall multiplicity.

The second part of the model is to convolve the results of these dimensionless simulations, first with the distribution of core masses, which yields the distributions of multiplicity, mass ratio and eccentricity, as a function of primary mass; and second with the distribution of core angular momenta, which yields the distributions of semi-major axis and period, again as a function of primary mass.

Using the observed distribution of core masses, and a distribution of core angular momenta which is consistent with the observations, our model is able to reproduce the observed IMF, the observed high multiplicity frequency of pre-Main Sequence stars, the observed distribution of separations, and - for long-period systems - the observed distributions of eccentricity and mass-ratio, provided we invoke $\mathcal{N}=4$ or 5 and $\sigma_{\log M}=0.6$.

We presume that for short-period systems the distributions of eccentricity and mass-ratio are modified by the dissipative effects of subsequent tidal interaction and competitive accretion; and that the reduced multiplicity frequency in the field, compared with young clusters, is the result of dynamical interactions between stars formed in different cores but the same cluster, following ring dissolution. Further numerical experiments are required to explore the consequences of such interactions.
\end{abstract}

Key words. binaries: general - methods: $N$-body simulations - methods: statistical - stars: formation

\section{Introduction}

One of the main problems in star formation is to explain the wide range of scales over which multiple systems form. The observed distribution of binary separations (e.g. Duquennoy \& Mayor 1991, hereafter DM91; Fischer \& Marcy 1992, hereafter FM92) extends from $10^{-2}$ AU to $10^{5}$ AU. The explanation for this large range of separations must lie in the details of the star formation process. In the field, the multiplicity of mature solar-mass stars is around 60\% (DM91); and in some star formation regions, the multiplicity of young solar-mass objects is at least $80 \%$, and possibly higher (Ghez et al. 1993; Reipurth \& Zinnecker 1993; Simon et al. 1992; Patience et al. 2002; Duchêne et al. 2004). This implies that the genesis of multiple systems is an intrinsic part of the star formation process. A prestellar core is presumed to collapse and fragment to produce a dense ensemble of protostars. Interactions between these protostars and the ambient gas then determine their final masses, which ones end up in multiple systems, and their orbital parameters.
Understanding how cores fragment and how many objects are produced requires 3-D hydrodynamical simulations, which are (a) very computer intensive, and (b) highly dependent on the input physics and initial conditions of the cores (e.g. Tohline 2002). Few simulations can be performed, and the statistical properties of the resulting protostars are therefore poorly constrained. Also, such simulations are not able to follow star formation to completion, so the final fate of a star-forming core and the stars it produces is not completely resolved. In particular, the orbital parameters of the resulting multiple systems are unlikely to have reached their final values when a 3-D hydrodynamic simulation is terminated.

\subsection{Previous $N$-body work}

A complementary approach to hydrodynamic simulations is to use an $\mathcal{N}$-body code to follow the ballistic evolution of a system of protostars, treating them as point masses, and thereby ignoring complicated gas-dynamical processes like fragmentation, merger and accretion. This approach has been 
pioneered by Kroupa and co-workers (Kroupa 1995a,b; Kroupa \& Bouvier 2003a,b; Kroupa et al. 2003), and by Sterzik \& Durisen (1998, 2003), with a view to explaining the observed statistics of binary stars. The work of Sterzik \& Durisen is similar to the present study, in that it is mainly concerned with the origin of the primordial binary properties in small- $\mathcal{N}$ clusters (or subclusters) of protostars. In Kroupa's work the emphasis is more on how these primordial binary properties are altered by subsequent dynamical interactions with other multiple systems and single stars in a wider cluster environment.

Sterzik \& Durisen (1998) investigate the binary statistics resulting from the dynamical dissolution of small- $\mathcal{N}$ clusters. They pick cluster masses from a power-law core mass spectrum, and calculate cluster radii from a scaling law of the form $R_{\text {CLUTER }} \propto M_{\text {CLUSTER }}^{\xi}(\xi=0,1$ or 2$)$. Each cluster contains $\mathcal{N}$ stars $(\mathcal{N}=3,4$ or 5$)$ with masses picked from a prescribed stellar mass spectrum. Initially the stars are positioned randomly in the cluster volume, with zero velocity. Their ballistic evolution is then followed for many crossing times, using an $\mathcal{N}$-body code, and the properties of the resulting multiple systems are recorded. However, this model is unable to reproduce the broad distribution of binary semi-major axes observed by DM91.

In a second paper Sterzik \& Durisen (2003) repeat these experiments, but now with clusters which initially are oblate and have some rotation about their short axis (specifically, $\beta=$ 0.1 , where $\beta$ is the ratio of rotational to gravitational energy). In addition, they relax the assumption of constant $\mathcal{N}$. Instead stars are chosen from a prescribed mass distribution until their total mass adds up to the preordained mass of the cluster, and this then determines $\mathcal{N}$ for that cluster. They are able to reproduce the dependence of multiplicity frequency on primary mass, but the distribution of semi-major axes is still much narrower than that observed by DM91.

Delgado-Donate et al. (2003) adopt a somewhat different approach, designed to capture hydrodynamic effects. They model a uniform-density core of isothermal gas using Smoothed Particle Hydrodynamics, and place 5 sink particles (representing protostellar embryos) at random positions within the core. Initially each sink contains only $2 \%$ of the total mass, but subsequently the sinks grow by competitive accretion and interact dynamically with one another, to generate a mass function which is a good fit to the observed Initial Mass Function. The resulting binary systems also have the right distribution of eccentricities. However, the distribution of semi-major axes is again much narrower than that observed by DM91, and is more similar to that observed in open clusters (Patience et al. 2002).

Kroupa (1995a) explores the evolution of a cluster of binary systems, and concludes that the properties of G-dwarf binaries in the field can be reproduced by dynamical interactions between binaries in a young cluster. Specifically he identifies a dominant mode cluster, which starts off with 200 binaries formed by random pairings from the field IMF, and with periods drawn randomly from a distribution covering 1 day to $10^{9}$ days and having no correlation with primary mass. Interactions amongst the binaries (termed stimulated evolution) reduces the binary fraction to $\sim 60 \%$, by selectively removing low-mass companions (i.e. dynamical biasing), and thereby reproduces the distributions of period and mass ratio observed in field G-dwarf binaries by DM91.

In Kroupa (1995b), this model is extended to include the eigenevolution of short-period systems (for example, their tidal circularization). He shows that stimulated evolution does not change the distribution of orbital eccentricities significantly (so the observed distribution must be essentially primordial). In addition, stimulated evolution does not generate sufficient higher multiples by capture to match the observed numbers of triples and quadruples. However, it does produce a distribution of mass ratios for G-dwarf primaries which is in good agreement with the observations of DM91. Kroupa (1995b) also suggests that long-period binaries with large eccentricities are somewhat more likely to be disrupted tidally than long-period binaries with low eccentricities.

Kroupa \& Burkert (2001) investigate the ballistic evolution of clusters comprising 100 or 1000 primordial binaries, with a narrow range of initial separations. They find that even under the most favourable conditions, dynamical interactions between binaries cannot produce a distribution of separations as broad as that observed by DM91. The observed separation distribution must therefore be set principally by the properties of primordial binaries.

Kroupa et al. (2001) show that the Pleiades may have evolved from a cluster like the Orion Nebula Cluster (ONC), following loss of residual gas and simultaneous stimulated evolution. They point out that the primordial binary population in the ONC could have been very similar to the preMain Sequence binary population that is currently observed in Taurus-Auriga. Subsequent dynamical interactions in the dense cluster environment then changed it into what we see today.

Kroupa \& Bouvier (2003a) show that the basic model developed in Kroupa (1995a) can not only explain the high multiplicity fraction for pre-Main Sequence stars in Taurus-Auriga, but also the apparent paucity of brown dwarfs (relative to the ONC). However, the basic model is seemingly unable to explain the binary properties of brown dwarfs: it produces too many stars with brown dwarf companions, and too many wide star-BD and BD-BD systems (Kroupa et al. 2003). It is also unable to reproduce the number of brown dwarfs per star, unless brown dwarfs have different formation mechanisms from stars, and possibly even different formation mechanisms in different environments (e.g. embryo ejection, photoevaporation, etc.; Kroupa \& Bouvier 2003b).

\subsection{Motivation for a new model}

In simulations of core collapse which include rotation (e.g. Bonnell \& Bate 1994; Cha \& Whitworth 2003; Hennebelle et al. 2004), in particular those where instability against collapse is triggered impulsively, the core may overshoot centrifugal balance and then bounce to form a dense ring, which subsequently fragments into multiple protostars. In this paper, we investigate the consequences of assuming that this is the dominant mechanism by which a core breaks up into individual protostars. Specifically, we use an $\mathcal{N}$-body code to follow the ballistic evolution of protostars which are initially distributed 
on a ring, and record their final binary statistics. We scale the mass and radius of the ring to match the observed distributions of core mass, core radius, and core rotation; and we compare the resulting binary statistics with observation. Our approach is similar to that adopted by Sterzik \& Durisen $(1998,2003)$. The main difference - and the reason that we obtain a broader distribution of separations, comparable with what is observed - is that we consider clusters having a distribution of $\beta$-values (i.e. a range of rotation rates), whereas Sterzik \& Durisen (1998) considered clusters with no rotation, and Sterzik \& Durisen (2003) considered clusters with a universal $\beta(=0.1)$.

In Sect. 2, we review the observations of cores which provide the input parameters for our model. In Sect. 3 we review the observations of stars and binary systems which our model seeks to explain. In Sect. 4 we sketch the sequence of stages through which the model is developed. In Sect. 5, we present the results of dimensionless simulations of ring dissolution. In Sect. 6, we scale the dimensionless results by convolving them with the core mass spectrum and obtain the distributions of multiplicity, eccentricity and mass ratio as a function of primary mass. In Sect. 7, we scale the results, by convolving them with the distribution of core angular momenta, and obtain the distribution of semi-major axes, as a function of primary mass. In Sect. 8, we summarize our main conclusions.

\section{Observations of cores}

In this section we review briefly the observations of cores which provide the input parameters for our model, namely the distribution of core masses (Sect. 2.1), the mass-radius relation for cores (Sect. 2.2), and the distribution of core rotation rates (Sect. 2.3).

\subsection{Core masses}

Motte et al. (2001) have measured the core-mass spectrum in Orion B and fitted it with a two part power law:

$$
\frac{\mathrm{d} \mathcal{N}_{\mathrm{CORE}}}{\mathrm{d} M_{\mathrm{CORE}}}= \begin{cases}k_{1} M_{\mathrm{CORE}}^{-1.5}, & M_{\mathrm{MIN}} \leq M_{\mathrm{CORE}} \leq M_{\mathrm{KNEE}} ; \\ k_{2} M_{\mathrm{CORE}}^{-2.5}, & M_{\mathrm{KNEE}} \leq M_{\mathrm{CORE}} \leq M_{\mathrm{MAX}},\end{cases}
$$

where $M_{\mathrm{MIN}}=0.5 M_{\odot}, M_{\mathrm{KNEE}}=1.0 M_{\odot}$ and $M_{\mathrm{MAX}}=10.0 M_{\odot}$. Similar results have been reported by Johnstone et al. (2001) for Orion B, by Motte et al. (1998) and Johnstone et al. (2000) for $\rho$ Ophiuchus, and by Testi \& Sargent (1998) for Serpens. We shall use Eq. (1) in our model but we extend the core mass spectrum up to $M_{\mathrm{MAX}}=20.0 M_{\odot}$.

\subsection{Core radii}

We shall assume that the initial core radii are given by the scaling relations

$R_{\mathrm{CORE}}\left(M_{\mathrm{CORE}}\right)=\left\{\begin{array}{cc}0.1 \mathrm{pc}\left(M_{\mathrm{CORE}} / M_{\odot}\right), & M_{\mathrm{CORE}}<M_{\odot} ; \\ 0.1 \mathrm{pc}\left(M_{\mathrm{CORE}} / M_{\odot}\right)^{1 / 2}, & M_{\mathrm{CORE}}>M_{\odot},\end{array}\right.$

(cf. Larson 1981; Myers 1983). Here, the low-mass regime applies to cores whose support is dominated by thermal pressure,

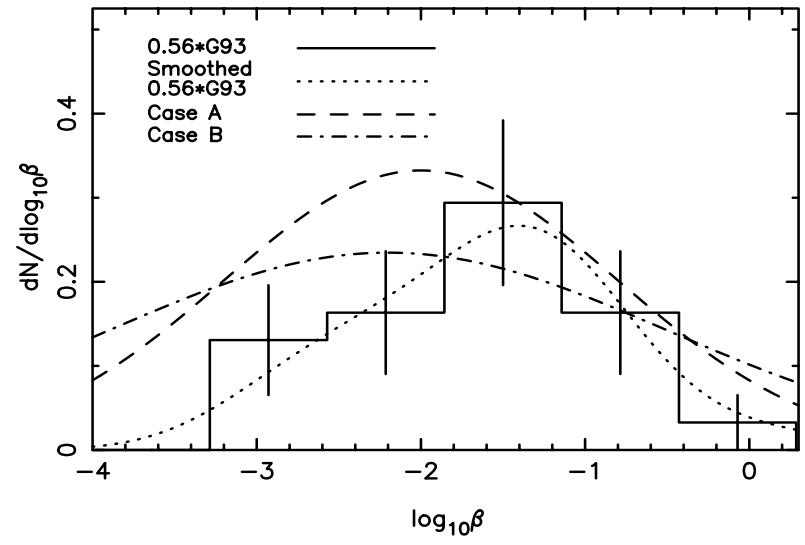

Fig. 1. The histogram and the dotted curve represent the distribution of observed $\beta$-values from Goodman et al. (1993), scaled to $56 \%$ (since they only measured rotation for 24 out of 43 cores). The dashed line represents the log-normal distributions of Case A $(\overline{\log \beta}=-2.0$, $\left.\sigma_{\log \beta}=1.2\right)$ and the dash-dot line represents the log-normal distribution of Case $\mathrm{B}\left(\overline{\log \beta}=-2.2, \sigma_{\log \beta}=1.7\right)$.

and the high-mass regime applies to cores whose support is primarily from turbulence. Strictly speaking there is a range of core radii at any given core mass, but since we are trying to keep the number of free parameters in the model to a minimum, we neglect this.

\subsection{Core rotation}

Goodman et al. (1993, hereafter G93) have measured the velocity profiles across a sample of 43 pre-stellar cores. They find statistically significant velocity gradients across many of the cores, and from these velocity gradients they calculate the ratio of rotational to gravitational potential energy, $\beta$, on the asumption of uniform global rotation, for 24 of the cores they observe. The distribution of these $\beta$-values, normalized to $24 / 43 \simeq 56 \%$, is represented by the histogram in Fig. 1, and also by the dotted line. The dotted line is obtained by smoothing the individual $\beta$ values with a Gaussian kernel having $\sigma_{\log \beta}=0.5$,

$$
\left.\frac{\mathrm{d} \mathcal{N}_{\mathrm{CORE}}}{\mathrm{d} \log \beta}\right|_{\mathrm{OBS}}=\sum_{i}\left\{\frac{1}{(2 \pi)^{1 / 2} \sigma_{\log \beta}} \exp \left[\frac{-\left(\log \beta_{i}-\log \beta\right)^{2}}{2 \sigma_{\log \beta}^{2}}\right]\right\},
$$

and is intended to mitigate the effects of arbitrarily binning a very small number of data points. However, due to low number statistics and the fact that only large projected velocity gradients can be measured, the full distribution of $\beta$-values is not well constrained by the observations of G93.

Burkert \& Bodenheimer (2000) have pointed out that the velocity gradients observed by G93 can also be explained by turbulent motions in cores, rather than uniform global rotation. By modelling the turbulence as a Gaussian random velocity field with power spectrum $P(k) \propto k^{-n}$ with $3 \leq n \leq 4$, they show that the resulting distribution of $\beta$-values fits the G93 observations well, and is approximately log-normal (see their Fig. 3, lower left panel). 

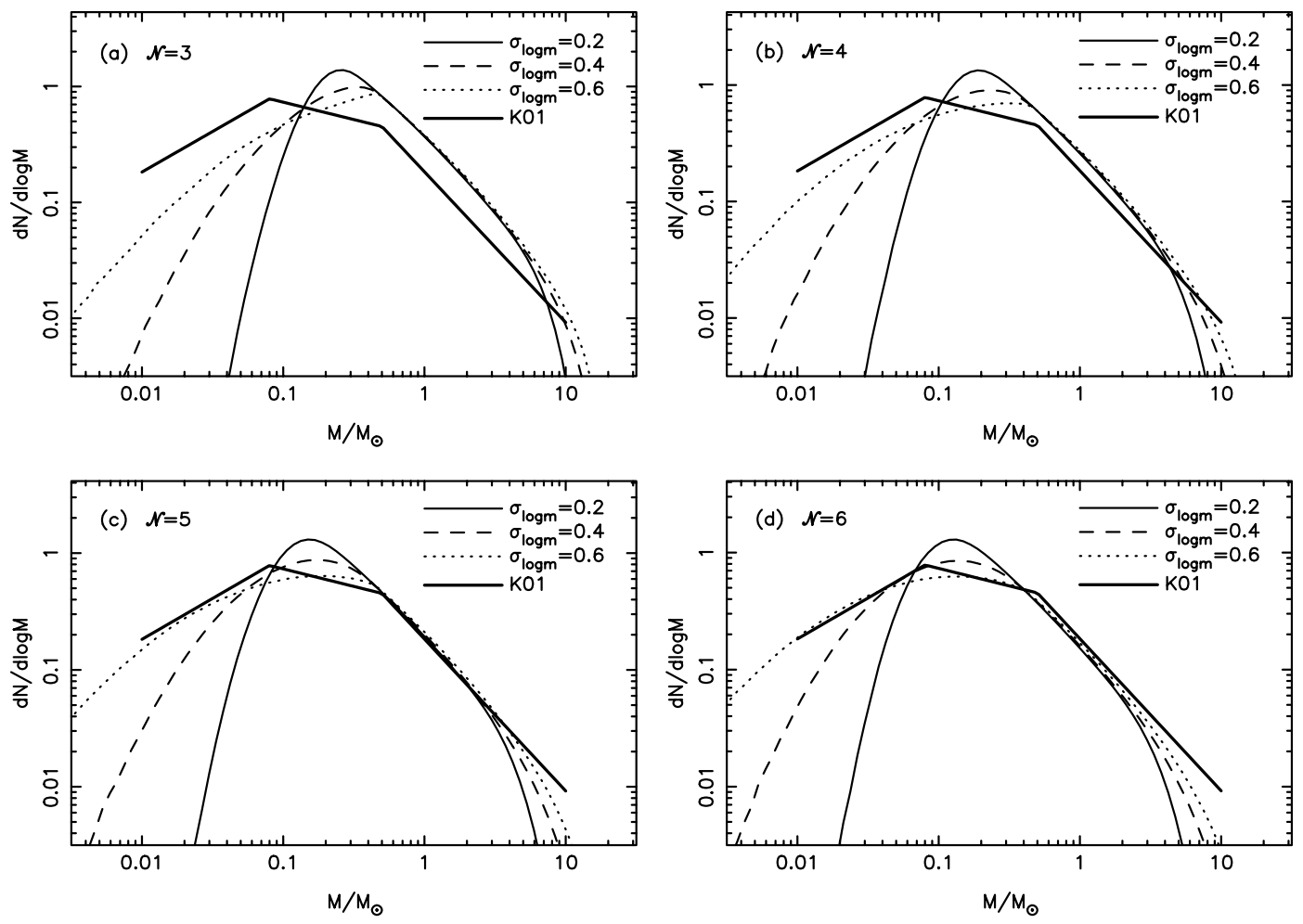

Fig. 2. a) The model IMF for $\mathcal{N}=3$ with $\sigma_{\log M}=0.2$ (thin solid line), 0.4 (dashed line) and 0.6 (dotted line); b) as a) but for $\mathcal{N}=4$; c) as a) but for $\mathcal{N}=5$; d) as a) but for $\mathcal{N}=6$. The observed IMF (Kroupa 2001) is shown as a heavy solid line on each panel.

Therefore we assume a $\log$-normal distribution of $\beta$-values:

$$
\frac{\mathrm{d} \mathcal{N}_{\mathrm{CORE}}}{\mathrm{d} \log \beta} \propto \exp \left(\frac{-(\log \beta-\overline{\log \beta})^{2}}{2 \sigma_{\log \beta}^{2}}\right) .
$$

Since $\mathrm{G} 93$ only determined $\beta$ for 24 of the 43 prestellar cores they observed, the observed $\beta$ distribution in Fig. 1 represents only $56 \%$ of all the cores, and we therefore have some freedom in choosing the parameters $\overline{\log \beta}$ and $\sigma_{\log \beta}$ for the full distribution in Eq. (4). We presume that some of the cores for which $\beta$ could not be determined were observed with inadequate resolution and/or from an unhelpful viewing direction (i.e. close to the angular momentum vector). However, this cannot account for all the non-determinations, and we assume that the majority of the non-determinations have $\beta$ values lower than those that are determined. Therefore we must invoke an overall distribution which contains the observed distribution, but extends to lower $\beta$ values. We consider two possibilities.

In Case $\mathrm{A}$ we adopt $\overline{\log \beta}=-2.0$ and $\sigma_{\log \beta}=1.2$ (dashed curve in Fig. 1). This is the less extreme possibility, in the sense that (a) it is easily compatible with the constraint of containing the observed distribution, and (b) it has most of the remaining $44 \%$ of $\beta$ values below, but only just below, the observed ones. It is therefore also our preferred possibility. In Sect. 7 we show that it yields a distribution of separations similar to that of the pre-Main Sequence binaries collated by Patience et al. (2002).

In Case B we adopt $\overline{\log \beta}=-2.2$ and $\sigma_{\log \beta}=1.7$ (dot-dash curve in Fig. 1). This is the more extreme possibility, in the sense that (a) it is only just compatible with the constraint of containing the observed distribution and (b) it has most of the remaining $44 \%$ of $\beta$ values not just below, but well below, the observed ones. In Sect. 7 we show that it yields a distribution of separations similar to that of the Main Sequence G-dwarf binaries in the field.

We assume that $\beta$ is not correlated with core mass, as indicated by G93 (their Fig. 13b).

\section{Observations of stars and binary systems}

In this section we review briefly the observations of stars and multiple systems which our model seeks to explain, namely the stellar Initial Mass Function (Sect. 3.1), stellar multiplicity (Sect. 3.2), the binary separation distribution (3.3), the binary eccentricity distribution (3.4) and the binary mass-ratio distribution (3.5). We stress that none of these distributions is used as input to the model. They are used solely for comparison with the predictions of the model.

\subsection{The Initial Mass Function (IMF)}

We will adopt the IMF determined by Kroupa (2001), i.e.

$\frac{\mathrm{d} \mathcal{N}_{\star}}{\mathrm{d} M_{\star}} \propto M_{\star}^{-\alpha} \mathrm{d} M_{\star}$,

where

$$
\begin{array}{ll}
\alpha_{0}=+0.3 \pm 0.7, & 0.01 M_{\odot} \leq M_{\star}<0.08 M_{\odot} ; \\
\alpha_{1}=+1.3 \pm 0.5, & 0.08 M_{\odot} \leq M_{\star}<0.50 M_{\odot} ; \\
\alpha_{2}=+2.3 \pm 0.3, & 0.50 M_{\odot} \leq M_{\star}<1.00 M_{\odot} ; \text { and } \\
\alpha_{3}=+2.3 \pm 0.7, & 1.00 M_{\odot} \leq M_{\star} .
\end{array}
$$

This IMF is plotted on all four panels of Fig. 2 (heavy solid lines). 

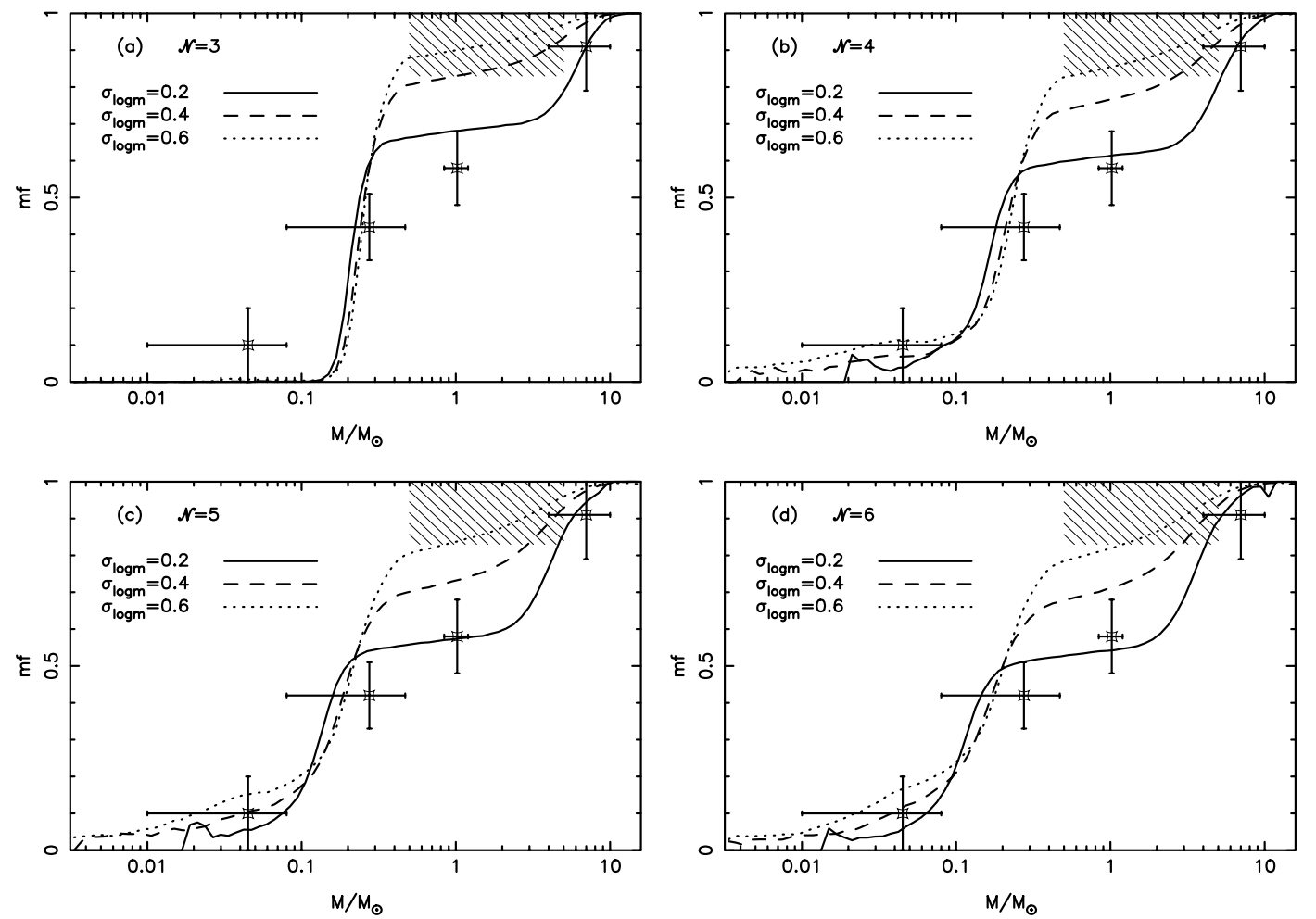

Fig. 3. The multiplicty frequency as a function of primary mass for a) $\mathcal{N}=3$, b) $\mathcal{N}=4$, c) $\mathcal{N}=5$, and $\mathbf{d}) \mathcal{N}=6$, all with $\sigma_{\log M}=$ 0.2, 0.4 and 0.6. The four plotted points with error bars are observational values taken from Martín et al. (2000), FM92, DM91 and Shatsky \& Tokovinin (2002). The hashed box represents the extrapolated multiplicty of PMS stars (Patience et al. 2002).

\subsection{Stellar multiplicity}

There are many different measures of multiplicity in use (e.g. Reipurth \& Zinnecker 1993; Goodwin et al. 2004b), but we will limit our discussion to the multiplicity frequency, $\boldsymbol{m} f$. If the total number of singles is $S$, the total number of binaries is $B$, the total number of triples is $T$, the total number of quadruples is $Q$, etc., then

$\boldsymbol{m f}=\frac{B+T+Q+\ldots}{S+B+T+Q+\ldots}$

and gives the fraction of systems which is multiple. $\boldsymbol{m} \boldsymbol{f}$ is more stable than the other measures, in the sense that it does not need to be revised if a multiple system is found to have additional components, only if a single has to be reclassified as a multiple.

Furthermore $\boldsymbol{m} \boldsymbol{f}$ can be defined as a function of primary mass,

$\boldsymbol{m} \boldsymbol{f}\left(M_{1}\right)=\frac{B\left(M_{1}\right)+T\left(M_{1}\right)+Q\left(M_{1}\right)+\ldots}{S\left(M_{1}\right)+B\left(M_{1}\right)+T\left(M_{1}\right)+Q\left(M_{1}\right)+\ldots}$,

where $S\left(M_{1}\right)$ is now the number of single stars of mass $M_{1}$, $B\left(M_{1}\right)$ is the number of binaries having a primary of mass $M_{1}$, and so on.

For Main Sequence stars, the observed values of $\boldsymbol{m} f\left(M_{1}\right)$ are $0.10 \pm 0.10$ for primaries in the mass range $\left(0.01 M_{\odot} \leq\right.$ $M_{1} \leq 0.08 M_{\odot}$ ) (Martín et al. 2000), $0.42 \pm 0.09$ for primaries in the mass range $\left(0.08 M_{\odot} \leq M_{1} \leq 0.47 M_{\odot}\right)$ (FM92), $0.58 \pm 0.10$ for primaries in the mass range $\left(0.84 M_{\odot} \leq M_{1} \leq\right.$ $1.20 M_{\odot}$ ) (DM91) and $0.91 \pm 0.12$ for primaries in the mass range $\left(4.0 M_{\odot} \leq M_{1} \leq 10.0 M_{\odot}\right)$ (Shatsky \& Tokovinin 2002). These observational points are plotted with error bars in Fig. 3.

For pre-Main Sequence (PMS) stars the situation is less clear, because observations of PMS binaries only cover a limited range of separations. However, in those separation ranges where PMS binaries can be observed, the multiplicity frequency appears to be significantly higher than for Main Sequence field stars in the same separation ranges. The compilation of Patience et al. (2002) suggests that for PMS primaries in the mass range $0.5 M_{\odot}<M_{1}<5 M_{\odot}$ the multipilicity frequency is in the range $0.83<\boldsymbol{m} \boldsymbol{f}<1.00$. This is shown as a hatched region in Fig. 3.

\subsection{The binary period and separation distributions}

DM91 have measured the binary properties of a complete sample of multiple systems in the solar neighbourhood having Main Sequence G-dwarf primaries. They find the distribution of periods to be approximately log-normal, i.e.

$\frac{\mathrm{d} \mathcal{N}_{\star}}{\mathrm{d} \log P_{\mathrm{d}}} \propto \exp \left\{-\frac{\left(\log P_{\mathrm{d}}-\overline{\log P_{\mathrm{d}}}\right)^{2}}{2 \sigma_{\log P}^{2}}\right\}$,

where $P_{\mathrm{d}}$ is the period in days and "log" is to the base 10 , $\overline{\log P_{\mathrm{d}}}=4.8$, and $\sigma_{\log P}=2.3$. The corresponding distribution of separations for multiple systems having Main Sequence G-dwarf primaries is then also log-normal (e.g. Ghez et al. 1993) with $\overline{\log a_{\mathrm{AU}}}=1.44$ and $\sigma_{\log a}=1.53$; Fig. $4 \mathrm{~b}$ illustrates this distribution. 

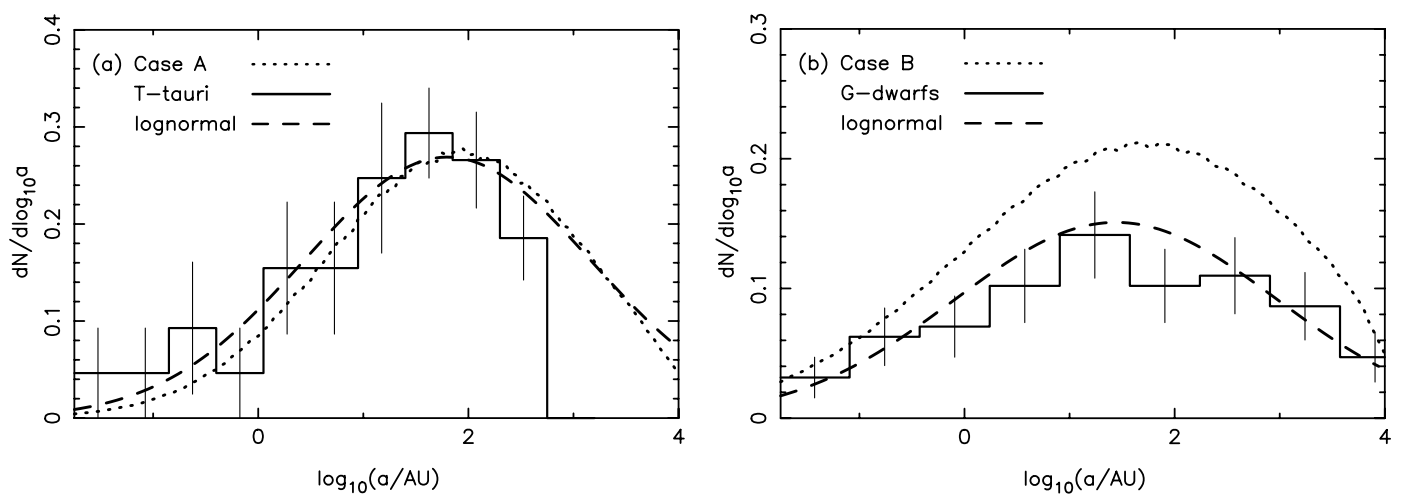

Fig. 4. a) The histogram shows the observed distribution of semi-major axes for pre-Main Sequence binaries in Taurus, as collated by Patience et al. (2002), and the dashed line is their best fit to this distribution. The dotted line shows the predictions of our model for Case $\mathrm{A}(\overline{\log \beta}=-2.0$ and $\sigma_{\log \beta}=1.2$ ) with parameters $\mathcal{N}=5$ and $\sigma_{\log M}=0.6$. The peak and width of the model predictions are fairly close to the observations, with a similarly high overall multiplicity. b) The histogram shows the observed distribution of semi-major axes for binaries with G-dwarf primaries in the field from DM91, and the dashed line is their log-normal fit to this distribution. The dotted line shows the predictions of our model for Case $\mathrm{B}\left(\overline{\log \beta}=-2.2\right.$ and $\left.\sigma_{\log \beta}=1.7\right)$ with parameters $\mathcal{N}=5$ and $\sigma_{\log M}=0.6$. The peak and width of the model predictions are similar to the observations, but the overall multiplicty is higher.

FM92 find a similar log-normal distribution of periods and separations for multiple systems in the solar neighbourhood having Main Sequence M-dwarf primaries.

Pre-Main Sequence binaries (Simon et al. 1992; Ghez et al. 1993; Reipurth \& Zinnecker 1993; Patience et al. 2002) have a distribution of separations that can again be approximated as log-normal over a limited range of separations, but it is different from that for Main-Sequence binaries in being shifted to larger separations, and somewhat narrower, with $\overline{\log a_{\mathrm{AU}}}=1.8 \pm 0.2$ and $\sigma_{\log a} \sim 1.36$ (Patience et al. 2002), as illustrated in Fig. 4a.

\subsection{The binary eccentricity distribution}

DM91 find that for binaries with Main Sequence G-dwarf primaries, the eccentricity distribution depends on the period. For long-period systems ( $P>10^{4}$ days, $\left.a>10 \mathrm{AU}\right)$, the eccentricty distribution is approximately thermal (i.e. $\mathrm{d} \mathcal{N}_{\star} / \mathrm{d} e=2 e$, Valtonen \& Mikkola 1991); the DM91 results for long-period binaries are shown in Fig. 8a. For short-period binaries with Main Sequence G-dwarf primaries $\left(P<10^{4}\right.$ days), the eccentricities tend to be significantly lower, with a distribution peaked around $e \simeq 0.2$. In addition, there appears to be an upper limit on the eccentricity, $e_{\mathrm{MAX}}(P)$, which decreases with decreasing $P$ and approaches zero for $P \lesssim 10$ days.

The data available for binaries with Main Sequence primaries of other spectral types (i.e. $\mathrm{M}$ dwarfs and $\mathrm{K}$ dwarfs) are limited, particularly for systems with long periods, but the overall distribution of eccentricity with period appears to be broadly similar to that for binaries with G-dwarf Main Sequence primaries. In particular, the upper limit on the eccentricity, $e_{\mathrm{MAX}}(P)$, decreasing with decreasing $P$ and approaching zero for $P \lesssim 10$ days, appears to apply to all Main Sequence binaries. This upper limit on $e$ is normally attributed to tidal circularization of close orbits.

For Pre-Main Sequence binaries the data on eccentricity is limited to short-period systems $\left(P<10^{4}\right.$ days $)$. Again there appears to be an upper limit on the eccentricity, $e_{\mathrm{MAX}}(P)$, which decreases with decreasing $P$ (Mathieu 1994). However, this limit is somewhat larger than that for binaries with G-dwarf Main Sequence primaries, and it only approaches zero for $P \lesssim 3$ days. Again, this is consistent with a picture in which close systems are circularized tidally; in pre-Main Sequence systems there has been less time for the process to work.

\subsection{The binary mass-ratio distribution}

The binary mass ratio, $q$, is defined by $q=M_{2} / M_{1}$, where $M_{1}$ is the primary mass, so necessarily $q \leq 1$.

DM91 find that for binaries having Main Sequence G-dwarf primaries, the distribution of $q$-values is dependent on the period. For long-period systems $\left(P>10^{4}\right.$ days $)$, the $q$-distribution has a significant peak at around $q=0.3$ (see Fig. 8b). For short-period systems, a much flatter distribution is observed, gently rising towards $q=1$ (Mazeh et al. 1992).

Mass ratios have also been determined for binaries having Main Sequence M-dwarf primaries by FM92. However, there are too few systems to reveal any clear dependence on period, and the sample is incomplete for $q<0.4$. For the whole sample, the distribution of mass ratios is consistent with being flat in the range $q \gtrsim 0.5$, but there is the suggestion of a decrease for lower $q$-values. We note that for M-dwarf primaries these low $q$-values ( $q \lessgtr 0.5$ ) usually correspond to brown dwarf companions.

Woitas et al. (2001) have determined mass ratios for preMain Sequence binaries with primary masses corresponding roughly to G- and M-dwarf Main Sequence stars. The mass ratios depend somewhat on whose evolutionary tracks are used to determine individual masses, but again the results are consistent with a flat distribution at large mass ratios $(q \gtrsim 0.5)$. There appears to be a decrease for $q \lesssim 0.3$, but this is probably due to incompleteness. 


\section{Outline of model}

\subsection{Assumptions and aims}

Our model of binary star formation is based on the assumption that all cores are rotating, that they collapse and fragment via ring formation, and that the resulting protostars then interact ballistically to form multiple systems. The aim of the paper is to investigate whether this simple model can explain the observed multiplicity of stars and their distributions of period, separation, eccentricity and mass-ratio.

\subsection{The model}

Consider a rotating prestellar core of mass $M_{\text {CORE }}$ which initially has radius $R_{\text {CORE }}$ and ratio of rotational to gravitational energy

$\beta=\frac{R_{\mathrm{CORE}}^{3} \Omega_{\mathrm{CORE}}^{2}}{3 G M_{\mathrm{CORE}}}=\frac{25 H_{0}^{2}}{12 G M_{\mathrm{CORE}}^{3} R_{\mathrm{CORE}}} \ll 1$.

If the core collapses conserving its angular momentum, $H_{0}$, and then bounces to form a centrifugally supported ring, the ring has radius

$R_{\mathrm{RING}} \simeq \beta R_{\mathrm{CORE}}$.

Suppose further that the ring is formed with approximately uniform line-density, but then fragments into $\mathcal{N}$ protostars (where we expect $\mathcal{N}$ to be small). Assume (i) that the $\mathcal{N}$ protostars formed from a single core have masses $M_{n}(n=1,2, \ldots, \mathcal{N})$ drawn from a log-normal distribution with standard deviation $\sigma_{\log M}$, and normalized so that

$\sum_{n=1}^{n=\mathcal{N}}\left\{M_{n}\right\}=f M_{\mathrm{CORE}}$,

where $f$ is the fraction of the core mass which is converted into stars (for simplicity we set $f=1$ here); (ii) that the protostars are initially distributed round the ring so that each protostar occupies a fraction of the circumference proportional to the protostar's mass; and (iii) that the protostars condense out sufficiently fast that we can follow their subsequent dynamics using pure $\mathcal{N}$-body methods.

For fixed $\mathcal{N}$ and $\sigma_{\log M}$, we first formulate the dynamical evolution in dimensionless form and simulate a large number of cases to obtain statistically robust distributions of (a) multiplicity, (b) orbital eccentricity, $e$, (c) component mass-ratio, $q \equiv M_{2} / M_{1}$, and (d) ratio of orbital separation to ring radius, $a / R_{\mathrm{RING}}$.

Then we convolve, first with the distribution of core masses, to obtain the overall stellar initial mass function (IMF) and the distributions of multiplicity, eccentricity and mass-ratio as a function of primary mass; and second with the distribution of core $\beta$-values to obtain the distribution of separations as a function of primary mass $M_{1}$.

The core mass spectrum is fairly tightly constrained by observation (Motte et al. 1998; Testi \& Sargent 1998; Johnstone et al. 2000; Motte et al. 2001; Johnstone et al. 2001), and therefore we do not adjust it (Sect. 2.1). Likewise the relation between core mass and core initial radius is constrained by observations, and we do not adjust it (Sect. 2.2). However, the distribution of $\beta$-values is less well constrained, and we consider two possible distributions (Sect. 2.3). $\mathcal{N}$ and $\sigma_{\log M}$ are treated as free parameters.

\section{Dimensionless simulations}

The first stage in constructing the model is to perform dimensionless simulations of low- $\mathcal{N}$ star clusters. The results of these simulations can later be scaled up to any mass and size depending on the given parameters of the core, i.e. $M_{\mathrm{CORE}}$ and $\beta$.

\subsection{Initial conditions}

We assume that a collapsing core forms a centifugally supported ring having radius $R_{\text {RING }}$ given by Eq. (11). However, for the dimensionless simulations we set $R_{\mathrm{RING}}=1$. There are then two free parameters which must be explored in the dimensionless simulations. First, we must specify the number of stars in the ring, $\mathcal{N}$. Second, as posited in Sect. 4.2, the stellar masses must be drawn at random from a log-normal distribution having standard deviation $\sigma_{\log M}$,

$$
\frac{\mathrm{d} \mathcal{N}_{\star}}{\mathrm{d} \log M}=\frac{\mathcal{N}}{(2 \pi)^{\frac{1}{2}} \sigma_{\log M}} \exp \left(\frac{-(\log M)^{2}}{2 \sigma_{\log M}^{2}}\right),
$$

and therefore we must specify $\sigma_{\log M}$. Strictly the mass distribution extends from $-\infty$ to $+\infty$ but for computational convenience we choose to curtail it at $\pm 3 \sigma_{\log M}$. Once the $\mathcal{N}$ stellar masses $M_{n}(n=1$ to $\mathcal{N})$ have been drawn randomally from this distribution, they are re-scaled by a factor $g, M_{n} \rightarrow \mu_{n}=g M_{n}$, so that the total system mass is equal to unity, i.e.

$\sum_{n=1}^{n=\mathcal{N}}\left\{\mu_{n}\right\}=1$

(We note that scaling the stellar masses in this way skews the overall distribution of masses slightly; it is no longer precisely log-normal. This is not a critical element of the model. The explanation is given in the Appendix.)

Next, we must specify the initial positions and velocities of the stars on the ring. If a ring having uniform line-density fragments into $\mathcal{N}$ stars, then each star $n$ forms from material in an "angular segment", $\Delta \theta_{n}$, proportional to it's mass, i.e.

$\Delta \theta_{n}=2 \pi \mu_{n}$

Thus in circular polar co-ordinates, we put $\theta_{1}=0$ and

$\theta_{n}=\theta_{n-1}+\frac{1}{2}\left(\Delta \theta_{n-1}+\Delta \theta_{n}\right), n=2,3, ., \mathcal{N}$.

In order to ensure conservation of linear momentum, each star $n$ must be placed at the centre of mass of the material from which it forms, i.e. at radius

$r_{n}=\frac{\sin \left(\mu_{n} \pi\right)}{\mu_{n} \pi}$

and then it must be given a circular speed

$v_{n}=V \frac{\sin \left(\mu_{n} \pi\right)}{\mu_{n} \pi}$ 
where $V$ must be chosen so that the system is virialized. Only part of the angular momentum of the initial ring goes into the orbital motion of the stars in the cluster. The remaining angular momentum must go into spin of the individual stars and their attendant discs, which are not modelled here.

\subsection{Numerical method}

The ring is then evolved balistically using an adapted version of NBODY3, supplied by Sverre Aarseth (e.g. Aarseth 1999). NBODY3 uses a fourth-order integrator, plus special regularisation routines, which are required for close encounters. 2-body regularisation is used to treat binaries and close encounters by transforming the co-ordinates of the binary components and calculating the motion separately from the rest of the system (e.g. Aarseth 2001). This technique reduces the error generated during close encounters and eliminates the need for extra terms such as gravity softening. Close interactions between three bodies or more can be treated using 3-body, 4-body and chain regularisation. Wider binaries and higher order multiple systems such as hierarchical triples and quadruples are identified by calculating the 2-body energies of all the pairs and selecting the most bound pair.

\subsection{Parameters}

Simulations are performed for all possible combinations of $\mathcal{N}=3,4,5$ and 6 , and $\sigma_{\log M}=0.2,0.4$ and 0.6 , and the distributions of multiplicity, semi-major axis (as a fraction of the radius of the ring), eccentricity, and mass-ratio are recorded.

For each set of parameters (i.e. each pair of $\mathcal{N}$ and $\sigma_{\log M}$ values), a large number of runs is required to obtain statistically significant distributions. Each set of runs treats $10^{5}$ stars in total (e.g. for $\mathcal{N}=4,2.5 \times 10^{4}$ runs are performed). Small- $\mathcal{N}$ systems usually dissolve in a few tens of crossing times (e.g. Van Albada 1968; Sterzik \& Durisen 1998), so, to ensure that the majority of systems have dissolved at the end of a simulation, each realisation is run for about 1000 crossing times. We choose a conservative tolerance parameter for the time step, to ensure accurate integration. Specifically we require that energy is conserved to 1 part in $10^{5}$ over the entire integration.

\subsection{Dissolution timescale}

Typically a cluster dissolves after a few tens of dynamical times (e.g. Van Albada 1968) leaving ejected singles and a variety of multiple systems, i.e. binaries, triples and quadruples. In the present context, we define the dynamical timescale of the initial ring to be $T_{\mathrm{RING}}=2 \pi\left(R_{\mathrm{RING}}^{3} / G M_{\mathrm{CORE}}\right)^{1 / 2}$; this is roughly equivalent to the crossing time for a cluster with isotropic velocity dispersion. Figure $7 \mathrm{a}$ shows the dissolution times, $T_{\text {DISS }}$, as a function of $T_{\mathrm{RING}}$, for the case $\mathcal{N}=5$ and $\sigma_{\log M}=0.6$. About half of the rings have dissolved after $20 T_{\mathrm{RING}}$, in agreement with previous numerical work.
Table 1. The numbers of different multiple systems produced in the dimensionless simulations.

\begin{tabular}{lcllll}
\hline \hline & & $\mathcal{N}=3$ & $\mathcal{N}=4$ & $\mathcal{N}=5$ & $\mathcal{N}=6$ \\
\hline$\sigma_{\log m}=0.2$ & $S$ & 33253 & 39711 & 43873 & 46567 \\
& $B$ & 33241 & 22506 & 20207 & 19306 \\
& $T$ & 88 & 5091 & 5014 & 4394 \\
& $Q$ & 0 & 1 & 168 & 409 \\
& $\boldsymbol{m} \boldsymbol{f}$ & 0.5001 & 0.4100 & 0.3666 & 0.3411 \\
\hline$\sigma_{\log m}=0.4$ & $S$ & 33231 & 41883 & 45534 & 48053 \\
& $B$ & 33228 & 22073 & 18033 & 16670 \\
& $T$ & 104 & 4657 & 5764 & 5389 \\
& $Q$ & 0 & 0 & 277 & 609 \\
& $\boldsymbol{m} \boldsymbol{f}$ & 0.5008 & 0.3896 & 0.3459 & 0.3205 \\
\hline$\sigma_{\log m}=0.6$ & $S$ & 33161 & 40950 & 41598 & 47707 \\
& $B$ & 33155 & 23098 & 16955 & 16378 \\
& $T$ & 176 & 4282 & 5645 & 5726 \\
& $Q$ & 0 & 1 & 333 & 589 \\
& $\boldsymbol{m} \boldsymbol{f}$ & 0.5013 & 0.4007 & 0.3554 & 0.3223 \\
\hline
\end{tabular}

\subsection{Multiplicity}

Table 1 shows the total numbers of singles, binaries, triples and quadruples produced and the multiplicity frequencies for different values of $\mathcal{N}$ and $\sigma_{\log M}$. As $\mathcal{N}$ is increased, $\boldsymbol{m} \boldsymbol{f}$ decreases. The reason for this is that a small cluster relaxes by dynamically ejecting stars, leaving a stable multiple system. For higher $\mathcal{N}$, more stars need to be ejected as singles before the cluster stabilizes, and this increases $S$, thereby reducing $\boldsymbol{m} \boldsymbol{f}$. We do not consider systems with very large $\mathcal{N}$, since such systems are found to produce too many singles to match the observations. Also hydrodynamical simulations suggest that ring fragmentation produces only a small numbers of fragments (e.g. Cha \& Whitworth 2003). The effect of changing $\sigma_{\log M}$ is quite small compared with changing $\mathcal{N}$, and is not monotonic.

\subsection{The dimensionless separation distribution}

In Figs. 6a and b, we see that the separation distribution has an approximately log-normal form, but for high- $\mathcal{N}$ there is an asymmetric tail stretching to large-separations.

If we fix $\sigma_{\log M}$ and increase $\mathcal{N}$ (Fig. 6a), the peak separation shifts to smaller values and the overall distribution becomes broader. Increasing $\mathcal{N}$ tends to increase the number of 3-body interactions which must occur before a stable multiple is formed, and therefore it tends to increase the binding energy of the surviving multiple, i.e. to decrease its semimajor axis. The greater number of 3-body interactions also produces a wider logarithmic range of final separations because it is a stochastic process (i.e. one binary system may be stabilized by just two mild interactions and another by four violent interactions).

If we now fix $\mathcal{N}$ and increase $\sigma_{\log M}$ (Fig. 6b), the separation distibution shifts to larger separations and becomes somewhat broader. This is because the number of 3-body interactions which occur before a stable multiple is formed is fixed by $\mathcal{N}$ and is therefore the same. However, for large $\sigma_{\log M}$, the two most massive stars which form the final binary contain 

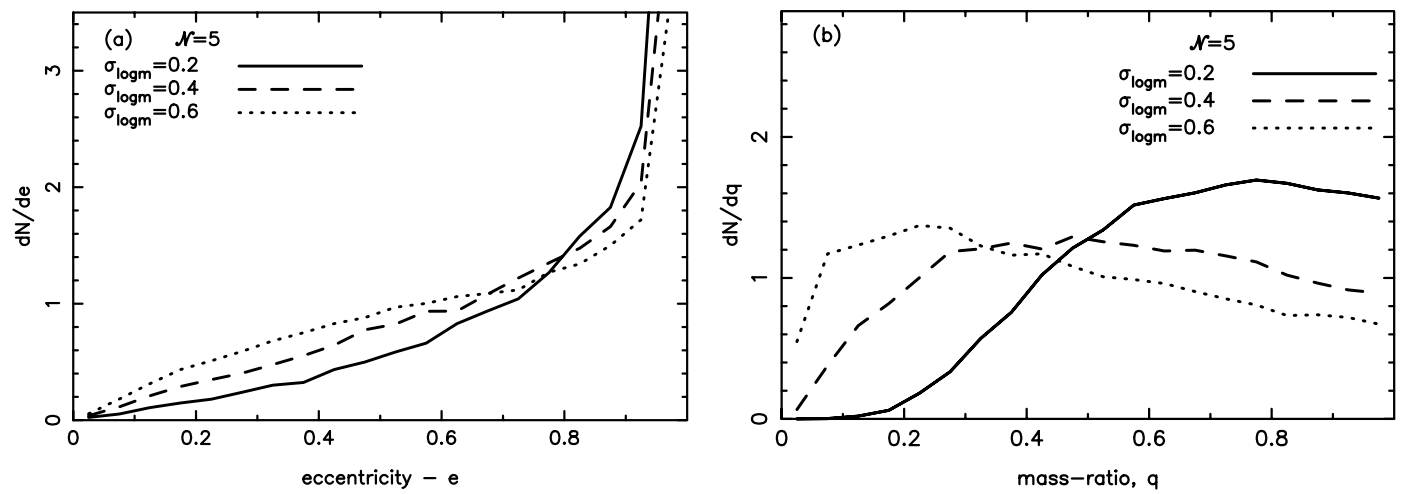

Fig. 5. The distributions of a) eccentricity and b) mass-ratio resulting from the dimensionless simulations. The parameters used are $\mathcal{N}=5$ and $\sigma_{\log M}=0.2$ (solid line), 0.4 (dashed line) and 0.6 (dotted line).
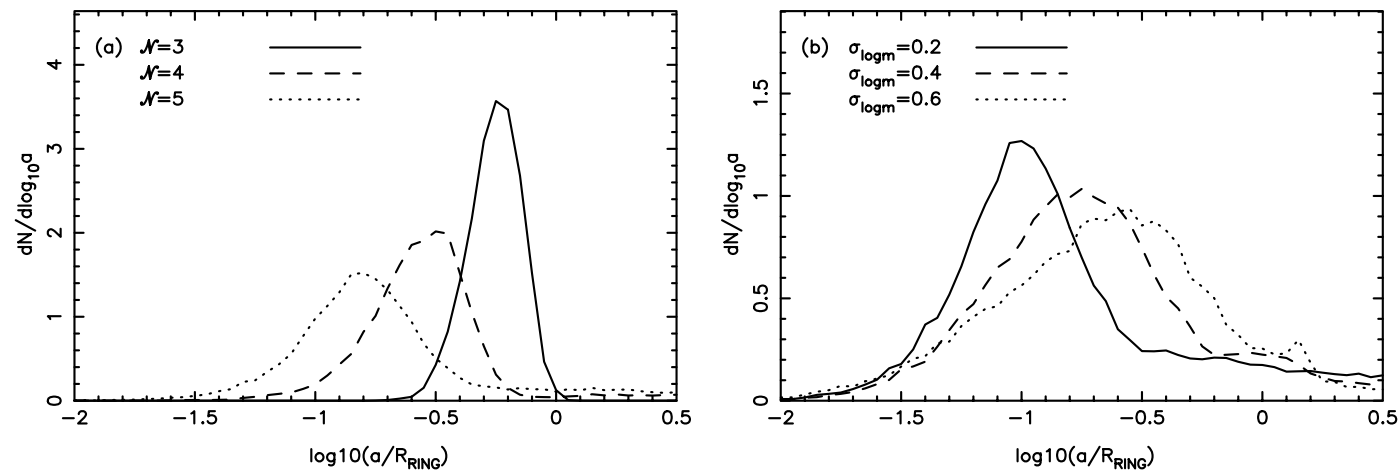

Fig. 6. The dimensionless separation distribution for clusters with a) $\mathcal{N}=3,4$ and 5 and $\sigma_{\log M}=0.2$, and b) $\mathcal{N}=6$ and $\sigma_{\log M}=$ $0.2,0.4$ and 0.6 .
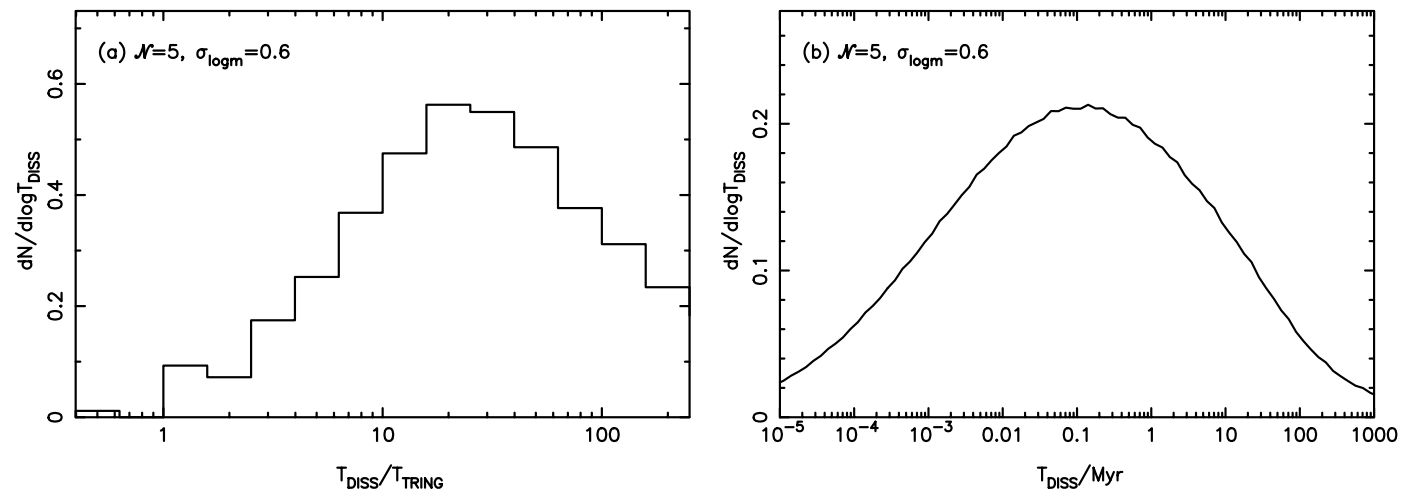

Fig. 7. a) Dimensionless lifetimes of small- $\mathcal{N}$ rings for $\mathcal{N}=5$ and $\sigma_{\log M}=0.6$. b) Lifetimes of all clusters with $\mathcal{N}=5$ and $\sigma_{\log M}=0.6$ when convolved over the core mass spectrum and the $\beta$ distribution in Myr.

almost all the mass, and the remaining stars are so lightweight, that their ejection does not harden the binary much. Conversely, for small $\sigma_{\log M}$ the two stars which form the final binary are of comparable mass to those which get ejected, and so their ejection hardens the remaining binary considerably.

\subsection{Eccentricities}

The distribution of eccentricities depends strongly on the value of $\sigma_{\log M}$, and much less on the value of $\mathcal{N}$. For low $\sigma_{\log M}$, there are few low-eccentricity binaries, and the numbers increase monotonically with increasing eccentricity, rising rapidly for very high eccentricities $(e>0.9)$. The rise at high eccentricities is characteristic of the dissolution of 2D planar systems; in contrast, the dissolution of 3D systems results in a thermal distribution of eccentricities $(\mathrm{d} \mathcal{N} / \mathrm{d} e=2 e$, e.g. Valtonen \& Mikkola 1991). As we increase $\sigma_{\log M}$, the number of high eccentricity binaries decreases and the distribution becomes flatter (but not flat, see Fig. 5a).

\subsection{Mass-ratios}

The distribution of mass-ratio, $q=M_{2} / M_{1}$, is found to be strongly dependent on $\sigma_{\log M}$, and only lightly dependent on $\mathcal{N}$. 
$\sigma_{\log M}$ controls the range of masses possible in a single core and thus the possible masses of components in a binary. If there is a low range of masses available, $q$ cannot differ greatly from unity. Figure $5 \mathrm{~b}$ shows that for $\operatorname{low} \sigma_{\log M}=0.2$, most of the binaries have mass-ratios greater than 0.5 with a peak at around $q=0.8$. As $\sigma_{\log M}$ is increased, there are more binaries with lower mass-ratios and the peak moves to a lower value of $q$.

\section{Convolving with the core-mass spectrum}

We can now convolve the dimensionless simulations with the core mass spectrum to produce an overall distribution of stellar masses (i.e. an IMF), the multiplicity as a function of primary mass $M_{1}$, and the distributions of eccentricity $e$ and massratio $q$, as functions of $M_{1}$.

\subsection{The resultant IMF}

First, we look at the IMFs generated by our model, and compare them with the observed IMF. The model IMFs, $\mathrm{d} \mathcal{N}_{\star} / \mathrm{d} M_{\star}$, are given by folding together the core mass function, $\mathrm{d} \mathcal{N}_{\mathrm{CORE}} / \mathrm{d} M_{\mathrm{CORE}}$ (Eq. (1)), and the dimensionless stellar mass spectrum, $\mathrm{d} \mathcal{N}_{\mu} / \mathrm{d} \mu$, as defined in Sect. 5.1,

$$
\frac{\mathrm{d} \mathcal{N}_{\star}}{\mathrm{d} M_{\star}}=\int_{M_{\mathrm{CORE}, \mathrm{MIN}}}^{M_{\mathrm{CORE}, \mathrm{MAX}}} \frac{\mathrm{d} \mathcal{N}_{\mu}}{\mathrm{d} \mu}\left(\mu=\frac{M_{\star}}{M_{\mathrm{CORE}}}\right) \frac{\mathrm{d} \mathcal{N}_{\mathrm{CORE}}}{\mathrm{d} M_{\mathrm{CORE}}} \frac{\mathrm{d} M_{\mathrm{CORE}}}{M_{\mathrm{CORE}}} .
$$

Figure 2 shows the IMFs for all combination of $\mathcal{N}=$ $3,4,5$ and 6 and $\sigma_{\log M}=0.2,0.4$ and 0.6. The shape of the IMF is highly dependent on $\sigma_{\log M}$. If we were to choose equal-mass stars (i.e. effectively $\sigma_{\log M}=0.0$ ), the IMF would exactly mimic the shape of the core mass spectrum. As we increase $\sigma_{\log M}$, the IMF becomes broader. The lowest mass core has mass $0.5 M_{\odot}$, so a large value of $\sigma_{\log M}$ is required to produce the large observed numbers of low-mass stars and brown dwarfs. $\mathcal{N}$ has little effect on the shape of the IMF, but affects the position of the peak. If we keep $\sigma_{\log M}$ constant and increase $\mathcal{N}$, the overall shape of the IMF is roughly constant, but the peak moves to smaller mass. We can fit the Kroupa IMF well with $\mathcal{N}=5$ and $\sigma_{\log M} \sim 0.6$; the ratio of brown dwarfs to stars is then $\sim 0.5$.

\subsection{Multiplicity}

Figure 3 shows the multiplicity frequency as a function of stellar mass, $\boldsymbol{m} \boldsymbol{f}\left(M_{1}\right)$, for all combination of $\mathcal{N}=3,4,5$ and 6 and $\sigma_{\log M}=0.2,0.4$ and 0.6. Overall, the model results have a similar trend to the observations, with $\boldsymbol{m} \boldsymbol{f}$ increasing from near zero at the lowest masses to near unity at the highest masses.

As $\sigma_{\log M}$ is increased, $\boldsymbol{m} \boldsymbol{f}$ increases at all masses above the peak in the initial mass function. This is because, as $\sigma_{\log M}$ is increased, these masses are increasingly likely to be the most massive star in the ring, and hence increasingly likely to form part of a multiple system due to dynamical biasing (McDonald \& Clarke 1993).

As $\mathcal{N}$ is increased, $\boldsymbol{m} \boldsymbol{f}$ decreases at all masses. This is because a small cluster evolves to a stable state (i.e. a binary), or a quasi-stable configuration (i.e. an hierarchical multiple), by ejecting stars. For higher $\mathcal{N}$, it is necessary to eject more stars before stability, or quasi-stability, is reached, and this decreases the overall multiplicity. If there is a range of masses, the lower mass stars are ejected preferentially.

Figure 3 shows that for intermediate stellar masses $\left(0.4 M_{\odot} \lesssim M_{*} \lesssim 4 M_{\odot}\right) \boldsymbol{m f}$ is almost independent of primary mass $M_{1}$. This is a direct consequence of using a simple powerlaw core mass function and convolving with a dimensionless distribution. For stars of given mass $M_{\star}$ in the range $0.4 M_{\odot}$ to $4 M_{\odot}$, there is an approximately constant ratio between the number of stars which have formed in a relatively low-mass core (and are therefore probably the most massive stars in that core and likely to end up as the primary in a multiple system) and the number of stars which have formed in a relatively highmass core (and are therefore probably one of the less massive stars in that core and unlikely to end up as a primary). This approximately constant ratio translates into an approximately constant $\boldsymbol{m} \boldsymbol{f}$.

For low masses $\left(M \lesssim 0.2 M_{\odot}\right), \boldsymbol{m} \boldsymbol{f}$ decreases rapidly with decreasing $M$. Low mass stars and brown dwarfs are therefore seldom found as primaries in binary systems, in agreement with observations. This decrease of $\boldsymbol{m} \boldsymbol{f}$ below $\sim 0.2 M_{\odot}$ would be less severe if the core mass spectrum were not cut off abruptly below $M_{\mathrm{MIN}}=0.5 M_{\odot}$ (see Eq. (1)).

If we consider the multiplicity of pre-Main Sequence stars, a high multiplicity $(>0.8)$ in the mass range $0.5 M_{\odot} \leq M_{1}<$ $5.0 M_{\odot}$ can only be realised in our model if $\sigma_{\log M}$ is large $(\sim 0.6)$. To obtain a good fit to the IMF, we require $\mathcal{N}=5$ and $\sigma_{\log M}=0.6$ (see Sect. 6.1). Therefore we can obtain a good fit to both the observed IMF, and the observed high $\boldsymbol{m f}$ for PMS stars, with $\mathcal{N}=5$ and $\sigma_{\log M}=0.6$.

However, there exists no parameter set that results in a satisfactory fit to the observed IMF, and reproduces the $\boldsymbol{m} \boldsymbol{f}$ of mature field stars. For example, to obtain a $\boldsymbol{m} \boldsymbol{f}$ of about 0.6 for G-dwarf primaries requires a low $\sigma_{\log M} \simeq 0.2$, but this produces a poor IMF which has too few brown dwarfs. This inconsistency can be resolved, if we retain $\sigma_{\log M}=0.6$ and the $\boldsymbol{m} \boldsymbol{f}$ for G-dwarf primaries is reduced, after ring dissolution, by the interactions which occur between binary systems formed in different rings. This is essentially what Kroupa (1995a) calls stimulated evolution.

\subsection{Eccentricity}

The eccentricity distribution of $\mathrm{G}$ dwarfs in our model is consistent with the observed eccentricity distribution for longperiod systems (DM91; Fig. 8a). We expect that the eccentricities of short-period systems are modified by tidal forces between the two components and/or mass equalization by accretion (e.g. Whitworth et al. 1995; Bate 2000), neither of which processes is modelled in this work. Therefore we only compare our model to the observed eccentricity distribution for longperiod systems. The observations are fitted best by $\mathcal{N}=4$ or 5 and $\sigma_{\log M}=0.4$ or 0.6 (see Fig. 8a).

For all cases, there is an excess of binaries with very high eccentricity $(e>0.9)$ compared to the observations. The simulations of Delgado et al. (2003) show that these systems 

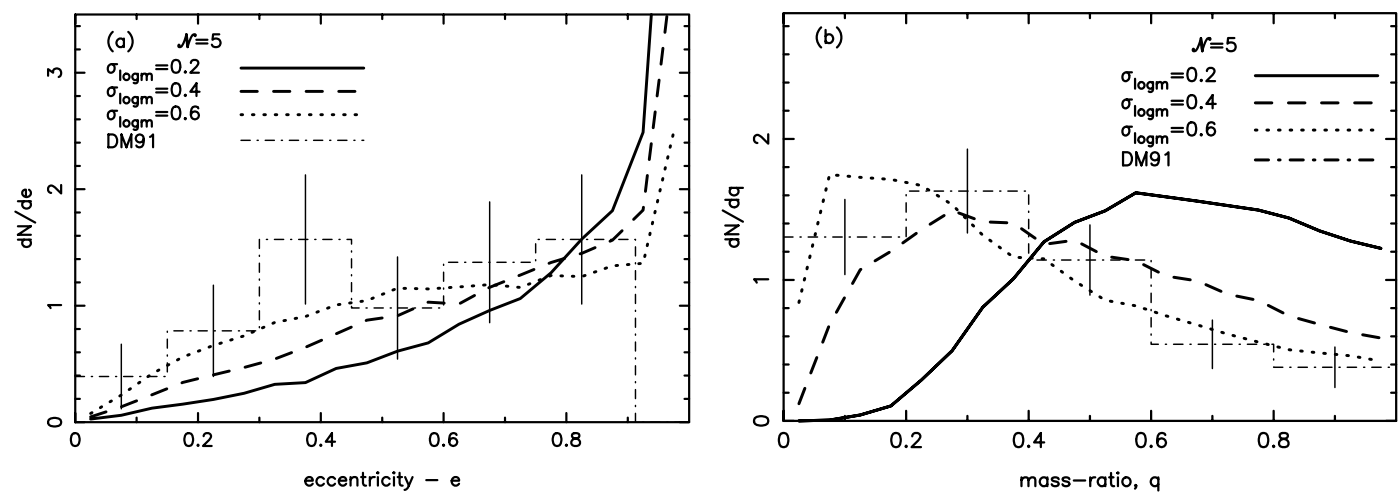

Fig. 8. The distributions of a) eccentricity and b) mass-ratio for the long-period G-dwarf binaries observed by DM91 (dot-dashed histogram, are compared with our model results. The model parameters used are $\mathcal{N}=5$ and $\sigma_{\log M}=0.2$ (solid line), 0.4 (dashed line) and 0.6 (dotted line).

would migrate to lower eccentricities if proper account were taken of gas dynamical processes at periastron during the protostellar stage (tidal interactions, mass exchange, etc.). Higheccentricity systems may also be more susceptible to tidal disruption, as noted by Kroupa (1995b).

\subsection{Mass-ratio}

The mass-ratio distribution from our model, with $\mathcal{N}=4$ or 5 and $\sigma_{\log M}=0.4$ or 0.6 , is very similar to the mass-ratio distribution for G dwarfs observed by DM91 (as illustrated in Fig. 8b), but somewhat different from the mass ratio distribution observed in Taurus by Woitas et al. (2001). This difference may be attributable to Taurus having an unusual IMF, and in particular a paucity of brown dwarfs (Briceño et al. 2002; Luhman et al. 2003). However, the evidence for an unusual IMF in Taurus has recently been called into question (Luhman 2004; Kroupa et al. 2003), and it may be necessary to seek another explanation for this difference.

\section{Convolving with the distribution of core rotation rates}

\subsection{The binary separation distribution}

In order to obtain the distribution of separations, we must convolve the dimensionless results with both the distribution of core masses $M_{\mathrm{CORE}}$, and the distribution of core $\beta$-values. $M_{\mathrm{CORE}}$ gives the core radius $R_{\text {CORE }}$ through Eq. (2), and $\beta$ gives the ring radius $R_{\text {RING }}$ through Eq. (11). Knowing $M_{\text {CORE }}$ and $R_{\text {RING }}$, the dimensionless simulations can be scaled to give the distributions of $a$ and $P$, as a function of primary mass $M_{1}$. As explained in Sect. 2.3 we consider two different $\beta$ distributions.

Using Case A $\left(\overline{\log \beta}=-2.0, \sigma_{\log \beta}=1.2\right)$, our model gives the distribution of semi-major axes illustrated in Fig. 4a (dotted line), where it is compared with the observations of pre-Main Sequence binaries having primary mass in the range $0.5 M_{\odot} \leq M_{1}<5.0 M_{\odot}$ collated by Patience et al. (2002; dashed line and histogram). We see that there is close corresondence between these two distributions. The implication is that, if cores have a distribution of rotation rates similar to the one adopted in Case A, then the dynamical dissolution of small rings of protostars is able to reproduce the distribution of binary periods observed for pre-Main Sequence stars like those in Taurus and Ophiuchus.

Using Case $\mathrm{B}\left(\overline{\log \beta}=-2.2, \sigma_{\log \beta}=1.7\right)$, our model gives the distribution of semi-major axes illustrated in Fig. 4b (dotted line), where it is compared with the observations of G Dwarf binaries in the field by DM91 (dashed line and histogram). We see that there is a very close correspondence between the two distributions. The implication is that, if cores have a distribution of rotation rates similar to the one adopted in Case B, then the dynamical dissolution of small rings of protostars is able to reproduce the distribution of binary periods observed for G-Dwarf binaries in the field by DM91. The problem is that if we persist with the parameters $\mathcal{N}=5$ and $\sigma_{\log M}=0.6$, the resulting multiplicity fraction for G-dwarf binaries is much higher than observed, as noted in Sect. 6.2.

However, if most of the stars in the field are formed in populous clusters, we must allow that their binary statistics continue to evolve dynamically after the dissolution of the rings in which they are formed. The cause of further dynamical evolution is that, following dissolution of the individual rings, the stars and binary systems formed in one ring interact with the stars and binary systems formed in other neighbouring rings. In other words, the individual rings are just subclusters within the larger cluster, and interactions on the scale of the cluster also influence the final binary statistics. Kroupa (1995a) has shown that interactions between binaries in clusters can widen the distribution of binary separations somewhat, and reduce the multiplicity from near unity to that observed in the field.

\subsection{Dissolution timescales}

The dimensionless ring dissolution times, $T_{\text {DISS }} / T_{\text {RING }}$, derived in Sect. 5.4, can be converted into physical times by combining Eqs. (2) and (11) with $T_{\text {RING }}$ (Sect. 5.4) to obtain

$\left(\frac{T_{\mathrm{DISS}}}{\mathrm{Myr}}\right)= \begin{cases}2.8\left(\frac{T_{\mathrm{DISS}}}{T_{\mathrm{RING}}}\right)\left(\frac{M_{\mathrm{CORE}}}{M_{\odot}}\right) \beta^{3 / 2}, & M_{\mathrm{CORE}} \leq M_{\odot} ; \\ 2.8\left(\frac{T_{\mathrm{DISS}}}{T_{\mathrm{RING}}}\right)\left(\frac{M_{\mathrm{CORE}}}{M_{\odot}}\right)^{1 / 4} \beta^{3 / 2}, & M_{\mathrm{CORE}} \geq M_{\odot} .\end{cases}$

Hence by convolving the distribution of $T_{\text {DISS }} / T_{\text {RING }}$ (Fig. 7) with the core mass spectrum (Eq. (1)) and the $\beta$-distribution 
(Eq. (4)), we obtain the overall distribution of dissolution times for the ring-clusters invoked in our model (Fig. 7b). The distribution peaks at around $\sim 0.2 \mathrm{Myr}$, the majority of the ring- clusters has dissolved by $1 \mathrm{Myr}$, and only a handful $(<3 \%)$ remains after 100 Myr.

\section{Conclusions}

We have developed a model of binary star formation in which cores collapse and bounce to produce rings, and the rings then fragment into protostars. The dynamical evolution of the protostars is followed using Aarseth's NBODY3 code, for many different realizations, and the properties of the resulting multiple systems are recorded.

We adopt (i) the observed distribution of core masses; (ii) the observed scaling relation between core mass and core radius; and (iii) two possible log-normal distribution of core rotation (Case A and Case B), which are consistent with the limited observations of core rotation, but attempt to take account of the selection effects which make the rotation of some cores unmeasurable (see Fig. 1). Case $\mathrm{A}\left(\overline{\log \beta}=-2.0, \sigma_{\log \beta}=1.2\right)$, which is our preferred option, assumes that the tendency to detect higher rotation rates is small, i.e. most of the undetected rotation rates are not much smaller than the detected ones. In contrast, Case $\mathrm{B}\left(\overline{\log \beta}=-2.2, \sigma_{\log \beta}=1.7\right)$ assumes that the tendency to detect higher rotation rates is large, i.e. the undetected rotation rates are usually much smaller than the detected ones. We stress that this dichotomy of model rotation rates is unavoidable, because of the incompleteness of the observed rotation rates, but it only affects the distribution of binary separations and the time-scale on which ring dissolution takes place.

Irrespective of the distribution of core rotation rates, our model reproduces the observed IMF (Fig. 2), and the distributions of eccentricity and mass ratio in long-period binary systems (Fig. 8), provided only that (a) each ring spawns $\mathcal{N} \sim 5$ protostars, and (b) the protostars have a log-normal mass-distribution with standard deviation $\sigma_{\log M} \sim 0.6$. Thus $\mathcal{N} \sim 5$ and $\sigma_{\log M} \sim 0.6$ are our preferred choices for these free parameters. The distributions of eccentricity and mass ratio for short-period binary systems are not reproduced by our model, but we presume that this is because our model does not include tidal circularization or mass equalization by accretion.

The model also reproduces the observed variation of multiplicity with primary mass for mature field stars (Fig. 3), but only if $\sigma_{\log M} \sim 0.2$. With our preferred values, $\mathcal{N} \sim 5$ and $\sigma_{\log M} \sim 0.6$, the model produces multiplicities which are higher than those observed for mature field stars, but are very similar to those observed for pre-Main Sequence stars, in the mass range 0.5 to $5 M_{\odot}$ (this is the only mass range for which reliable pre-main Sequence multiplicities are available). We conclude that the multiplicities resulting from ring dissolution represent the observed pre-main Sequence population well, and that the pre-main Sequence population then evolves into the observed Main Sequence population through interactions between stars and binary systems from different rings in the same cluster.

In a somewhat different context, Kroupa (1995a) has shown that such interactions can reduce the overall multiplicity from $\sim 1.0$ to $\sim 0.6$. Our model only requires such interactions to destroy $\sim 25 \%$ of pre-Main Sequence binaries, but a concern with the model is then that binaries with low mass-ratio will be destroyed preferentially (although not exclussively). This will skew the distribution of mass-ratios somewhat, and may thereby degrade the agreement with observations of mature G-dwarfs in the field. We are currently conducting numerical experiments to explore the effect of interactions between binary systems from different rings, in the cluster environment.

In order to predict the distribution of semi-major axes, we have to consider the distribution of core rotation rates. If we adopt Case A, our results match closely the distribution of semi-major axes obtained by Patience et al. (2002) for preMain Sequence stars (Fig. 4a), and we conclude that further dynamical evolution takes place - presumably involving interactions with protostars from other neighbouring rings - to convert this distribution into the one observed in the field.

Conversely, if we adopt Case B, our results match closely the peak and width of the separation distribution obtained by DM91 for field G Dwarfs (Fig. 4b). However, our results do not match the observed multiplicity frequency for field G-dwarfs, in the sense that our predicted $\boldsymbol{m} \boldsymbol{f}$-value is too high. It is unlikely that further dynamical evolution, following ring dissolution, can rectify this, since to preserve the peak and width of the separation distribution would require dynamical processes which destroy, with equal efficiency, binary systems having widely different separations. Therefore Case A is our preferred option.

Most rings have dissolved after $1 \mathrm{Myr}$ and fewer than 3\% remain after $100 \mathrm{Myr}$.

Acknowledgements. We would like to thank Simon Goodwin for many helpful discussions during this work, Gary Fuller for useful comments on the rotation of cores, and Sverre Aarseth for his NBODY3 code and patient help with learning to use it. We also thank the referee for comments which helped to improve the original version of the paper. D.A.H. gratefully acknowledges the support of a PPARC postgraduate studentship, and A.P.W. acknowledges the support of a European Commission Research Training Network awarded under the Fifth Framework (Ref. HPRN-CT-2000-00155).

\section{Appendix A: Skewing the log-normal distribution of stellar masses}

Stellar masses, $M_{n}(n=1, \mathcal{N})$ are initially picked randomly from a log-normal distribution which is symmetric in $\log M$ (Eq. (13)). These masses are then re-scaled by a factor $g$, $M_{n} \rightarrow \mu_{n}=g M_{n}$, so that the total mass is unity (Eq. (14)). After this re-scaling, the minimum possible mass is 0 , corresponding to $\log M=-\infty$, and the maximum possible mass is 1 , corresponding to $\log M=0$. It follows that the distribution can now only be symmetric in $\log M$ if its median lies at $\log M=-\infty$, but this is clearly non-sensical. In fact the distribution is skewed, in the sense of having a tail to low values of $\mu$, and the median is of order $-\log (\mathcal{N})$.

The asymmetry arises because we are invoking a finite - indeed small - number of stars. For simplicity consider the case $\mathcal{N}=2$, and assume that the two stars do not have equal mass. If the more massive star is initially (pre re-scaling) exceptionally 
massive, it has to be reduced to achieve $\sum\left\{\mu_{n}\right\}=1$, and this inevitably decreases the mass of the lower mass star, even if it was already quite small. Conversely, if the less massive star is initially of exceptionally low-mass, this has little or no influence on the re-scaling, which is mainly influenced by the more massive star. It is this asymmetry between the effects of exceptionally high-mass and exceptionally low-mass stars on the re-scaling that causes the re-scaled distribution to be skewed, particularly for small $\mathcal{N}$. The effect disappears as $\mathcal{N} \rightarrow \infty$.

\section{References}

Aarseth, S. J. 1999, PASP, 111, 1333

Aarseth, S. J. 2001, in The Restless Universe, ed. B. A. Steves, \& A. J. Maciejewski, IoP, 93

Bate, M. R. 2000, MNRAS, 314, 33

Bonnell, I. A., \& Bate, M. R. 1994, MNRAS, 271, 999

Briceño, C., Luhman, K. L., Hartmann, L., Stauffer, J. R., \& Kirkpatrick, J. D. 2002, ApJ, 580, 317

Burkert, A., \& Bodenheimer, P. 2000, ApJ, 543, 822

Cha, S.-H., \& Whitworth, A. P. 2003, MNRAS, 340, 91

Delgado-Donate, E. J., Clarke, C. J., \& Bate, M. R. 2003, MNRAS, 342,926

Duchêne, G., Bouvier, J., Bontemps, S., André, P., \& Motte, F. 2004, A\&A, 427, 651

Duquennoy, A., \& Mayor, M. 1991, A\&A, 248, 485 (DM91)

Fischer, D. A., \& Marcy, M. 1992, ApJ, 396, 178 (FM92)

Ghez, A. M., Neugebauer, G., \& Matthews, K. 1993, AJ, 106, 2005

Goodman, A. A., Benson, P. J., Fuller, G. A., \& Myers, P. C. 1993, ApJ, 406, 528 (G93)

Goodwin, S. P., Whitworth, A. P., \& Ward-Thompson, D. 2004, A\&A, 423, 169

Hennebelle, P., Whitworth, A. P., Cha, S.-H., \& Goodwin, S. P. 2004, MNRAS, 348, 687

Johnstone, D., Wilson, C. D., Moriarty-Schieven, G., et al. 2000, ApJ, 545,327

Johnstone, D., Fich, M., Mitchell, G. F., \& Moriarty-Schieven, G. 2001, ApJ, 559, 307
Kroupa, P. 1995a, MNRAS, 277, 1491

Kroupa, P. 1995b, MNRAS, 277, 1507

Kroupa, P. 2001, MNRAS, 322, 231

Kroupa, P., \& Bouvier, J. 2003a, MNRAS, 346, 343

Kroupa, P., \& Bouvier, J. 2003b, MNRAS, 346, 369

Kroupa, P., \& Burkert, A. 2001, ApJ, 555, 945

Kroupa, P., Aarseth, S., \& Hurley, J. 2001, MNRAS, 321, 699

Kroupa, P., Bouvier, J., Duchêne, G., \& Moraux, E. 2003, MNRAS, 346, 354

Larson, R. B. 1981, MNRAS, 194, 809

Luhman, K. L., Briceño, C., Stauffer, J. R., et al. 2003, ApJ, 590, 348

Luhman, K. L. 2004, ApJ, 617, 1216

McDonald, J. M., \& Clarke, C. J. 1993, MNRAS, 262, 800

Martín, E. L., Brander, W., Bouvier, J., et al. 2000, ApJ, 543, 299

Mathieu, R. D. 1994, ARA\&A, 32, 465

Mazeh, T., Goldberg, D., Duquennoy, A., \& Mayor, M. 1992, ApJ, 401, 265

Motte, F. A., André, P., \& Neri, R. 1998, A\&A, 336, 150

Motte, F. A., André, P., Ward-Thompson, D., \& Bontemps, S. 2001, A\&A, 372

Myers, P. C. 1983, ApJ, 1983, 270

Patience, J., Ghez, A. M., Reid, I. N., \& Matthews, K. 2002, AJ, 123, 1570

Reipurth, B., \& Zinnecker, H. 1993, A\&A, 278, 81

Shatsky, N., \& Tokovinin, A. 2002, A\&A, 382, 92

Simon, M., Chen, W. P., Howell, R. R., Benson, J. A., \& Slowik, D. 1992, ApJ, 384, 212

Sterzik, M. F., \& Durisen, R. H. 1998, A\&A, 339, 95

Sterzik, M. F., \& Durisen, R. H. 2003, A\&A, 400, 1031

Testi, L., \& Sargent, A. L. 1998, ApJ, 508, L91

Tohline, J. E. 2002, ARA\&A, 40, 349

Valtonen, M., \& Mikkola, S. 1991, ARA\&A, 29, 9

Van Albada, T. S. 1968, BAN, 19, 479

Whitworth, A. P., Chapman, S. J., Bhattal, A. S., et al. 1995, MNRAS, 277,727

Woitas, J., Leinert, Ch., \& Köhler, R. 2001, A\&A, 376, 982 